\begin{tabular}{|l|l|c|l|}
\hline Eiszeitalter u. Gegenwart & $\mathbf{4 5}$ & $42-58$ & Hannover 1995 \\
\hline
\end{tabular}

\title{
Die stratigraphische Stellung des Lößprofils von Mönchengladbach-Rheindahlen (Niederrhein)
}

\author{
Josef Klostermann \& Jürgen Thissen*)
}

Middle and Upper Pleistocene, Holocene, loess, soils, artefacts, geology, soilphysics, heavy mineral assoziation, pollenanalytical dates, tectonic events, stratigraphy, Mönchengladbach, Lower Rhine Embayment

\begin{abstract}
Kurzfassung: In der Ziegeleigrube Dreesen bei Mönchengladbach ist eine ca. $9 \mathrm{~m}$ mächtige Lößabfolge mit vier Parabraunerden aufgeschlossen. Die gesamte Schichtenfolge wurde an zwei ausgewählten Teilprofilen auf ihre Korngrößenzusammensetzung, das Porenvolumen, die Lagerungsdichte sowie den Schwermineral- und Polleninhalt untersucht. Zahlreiche Artefaktfunde erlauben eine stratigraphische Einstufung der Schichtenfolge. Sondierbohrungen in der näheren Umgebung machten Rückschlüsse auf tektonische Bewegungen im Mittel- und Oberpleistozän möglich. Es wird versucht, das Lößsprofil von Rheindahlen chronostratigraphisch einzuordnen.
\end{abstract}

\section{[The stratigraphic position of the loess profile near Mönchengladbach-Rheindahlen]}

\begin{abstract}
In the pit of the brickworks Dreesen near Mönchengladbach a $9 \mathrm{~m}$ thick loess sequence including four soils is outcropping. The whole sequence was investigated at two distinct profiles. There were carried out analysis of the grainsize, the porousity, the volumetric weight, the heavy mineral- and the pollencontent of the sediments. The stratigraphic correlation of the sediments could be carried out a multitude of artefacts. Borings carried out around the pit enabled us to draw conclusions from the tectonic movements during middle and upper Pleistocene time. It was tried ones hand at chronostratigraphical classification.
\end{abstract}

\section{Inhalt:}

1 Forschungsgeschichte

2 Das Westwandprofil der Ziegeleigrube Dreesen/Rheindahlen

2.1 Geologische und bodenphysikalische Daten

2.2 Schwermineralgesellschaften und Floren

2.3 Die Artefaktinventare

2.3.1 Fundschicht A1

2.3.2 Fundschicht A2/A3

2.3.3 Fundschicht B1/B2

2.3.4 Fundschicht B3

2.3.5 Fundschicht B4/B5

*) Anschriften der Verfasser: Priv.-Doz. Dr. Josef KLosterMANN, Geologisches Landesamt NRW, De-Greiff-Str. 195, 47803 Krefeld; Jürgen Thissen M. A., Hardter Waldstraße 101, 41169 Mönchengladbach

\author{
2.3.6 Fundschicht C1 \\ 3 Stratigraphische Einstufung der \\ Parabraunerden \\ 4 Versuch einer chronostratigraphischen \\ Einordnung \\ 5 Tektonische Bewegungen im Mittel- und \\ Ober-Pleistozän \\ 6 Schriftenverzeichnis
}

\section{Forschungsgeschichte}

In der Ziegeleigrube Dreesen im Südwesten von Mönchengladbach (s. Abb. 1) wird seit Beginn dieses Jahrhunderts Löß zur Ziegelherstellung abgebaut. Hier ist eine der wohl vollständigsten Lößabfolgen des Niederrheingebietes aufgeschlossen. Verschieden alte quartärzeitliche Lößaufwehungen sind dort durch mehrere, in der Regel vier, Parabraunerden voneinander getrennt. Parabraunerden entstehen während warmzeitlicher Klimaphasen. Gelingt es, die Parabraunerden stratigraphisch zuzuordnen, läßt sich ein Standardprofil für die niederrheinische Lößabfolge aufstellen. Der Löß wurde seit 1908 von Hand mit dem Spaten abgegraben. Seit 1936 geschah dies mit dem Eimerkettenbagger. Bei dieser Abbaumethode entstanden sehr glatte Profilwände, so daß Artefakte aus angeschnittenen Fundschichten sehr leicht im sonst steinfreien Sediment entdeckt werden konnten. Im Mai 1987 wurde der Abbau mit dem Eimerkettenbagger aus Rationalisierungsgründen aufgegeben und mit einem Löffelbagger fortgesetzt, was ein Auffinden von Steinartefakten in Zukunft nahezu unmöglich macht.

Schon 1915 erkannte BrockMEIER in Feuersteinen, die beim Lößabbau von den Arbeitern gefunden wurden, Werkzeuge des prähistorischen Menschen (BROCKMEIER 1929). In der folgenden Zeit wurden die Fundschichten vom Hangenden zum Liegenden hin mit Buchstaben versehen (Abb. 4). Die Artefakte aus dem Weichsellöß wurden den Fundschichten A1 - A3 zugewiesen. Die Funde zwischen der zweiten und dritten Parabraunerde mit B, die unter der dritten Parabraunerde mit $\mathrm{C}$ und die unterste mit $\mathrm{D}$ be- 


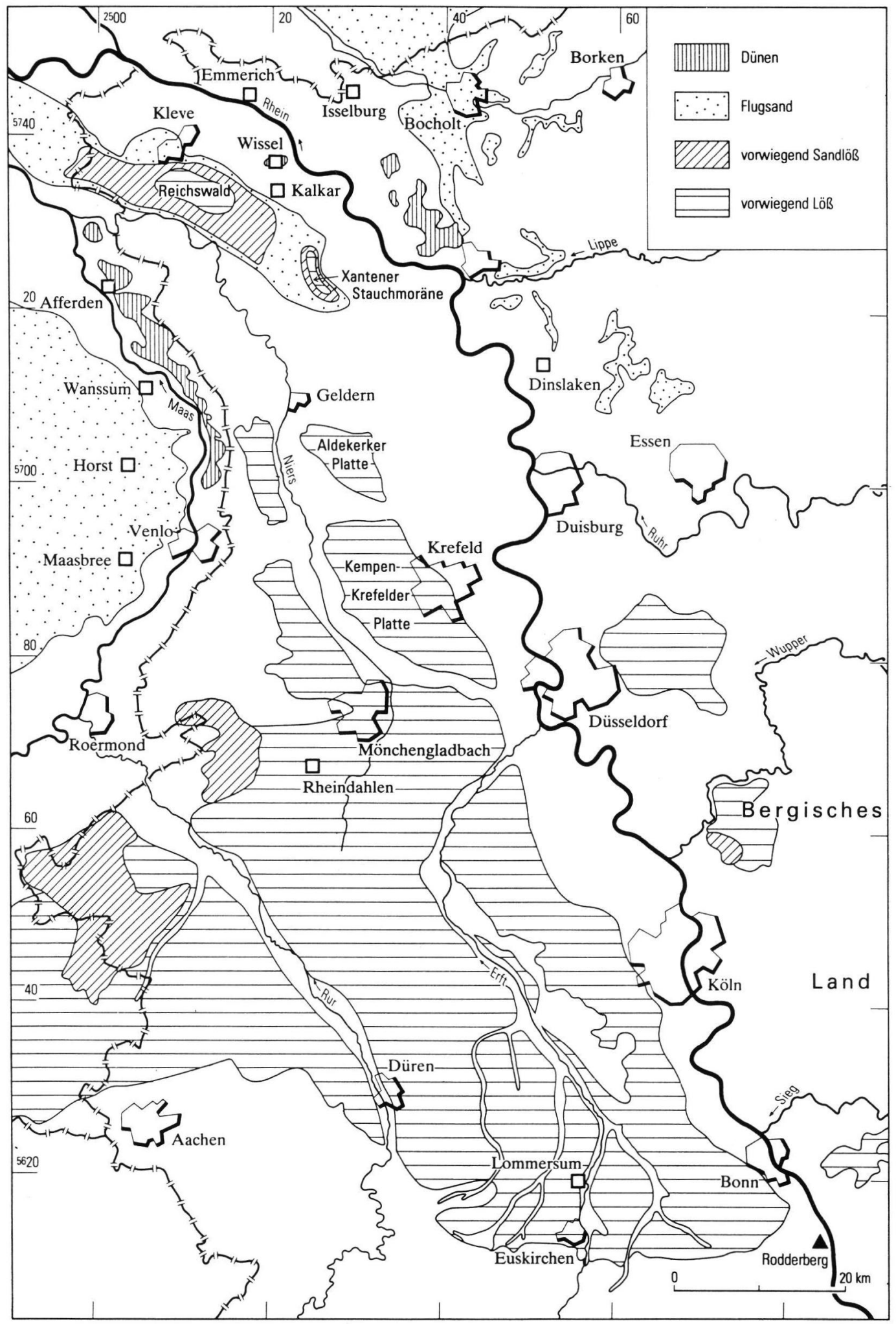

Abb. 1: Äolische Sedimente in der Niederrheinischen Bucht.

Fig. 1: Aeolian sedimentation in the Lower Rhineland. 


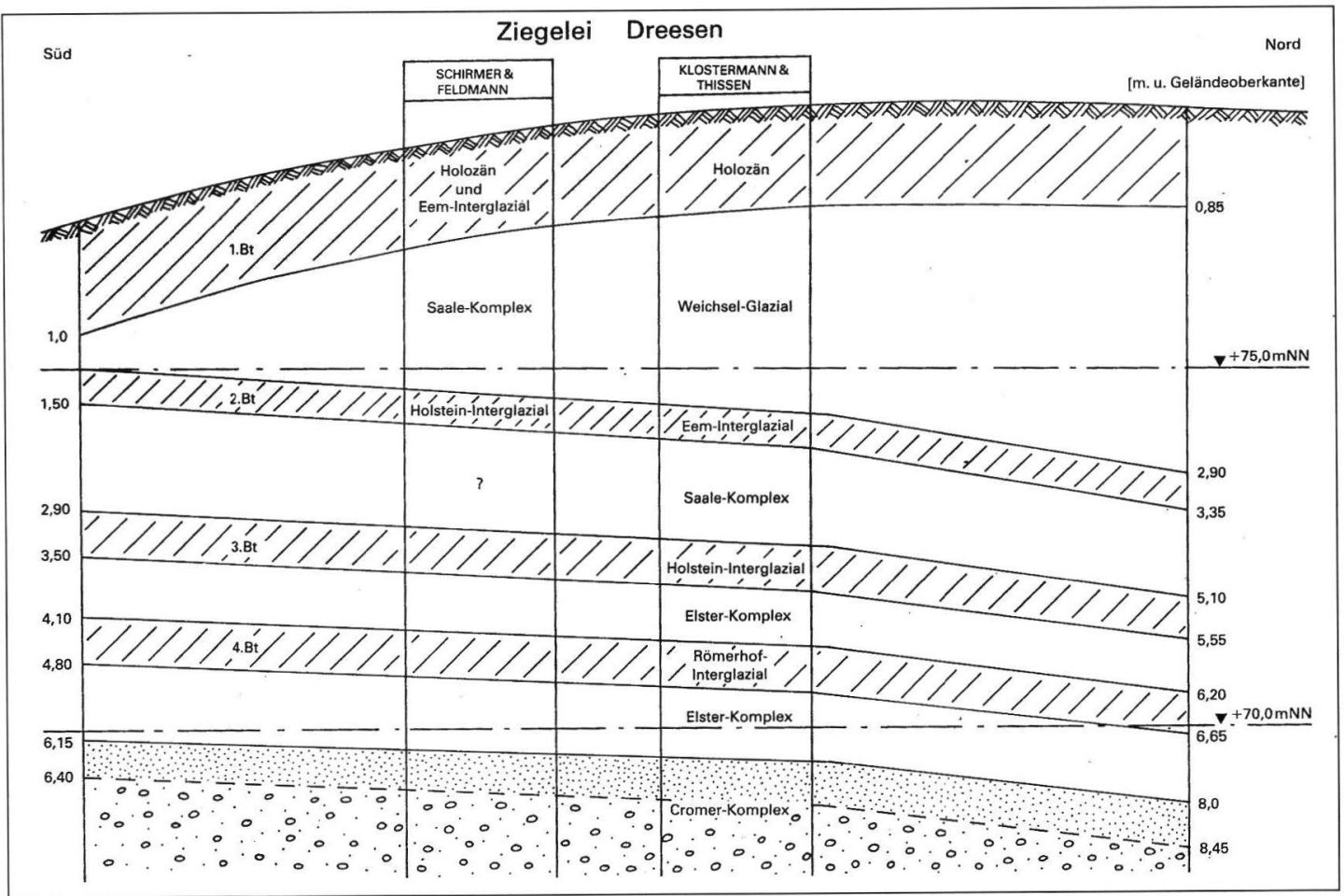

Abb. 2: Schematisches Profil der Rheindahlener Lößablagerungen.

Fig. 2: Schematic profile of the Rheindahlen loess deposits.

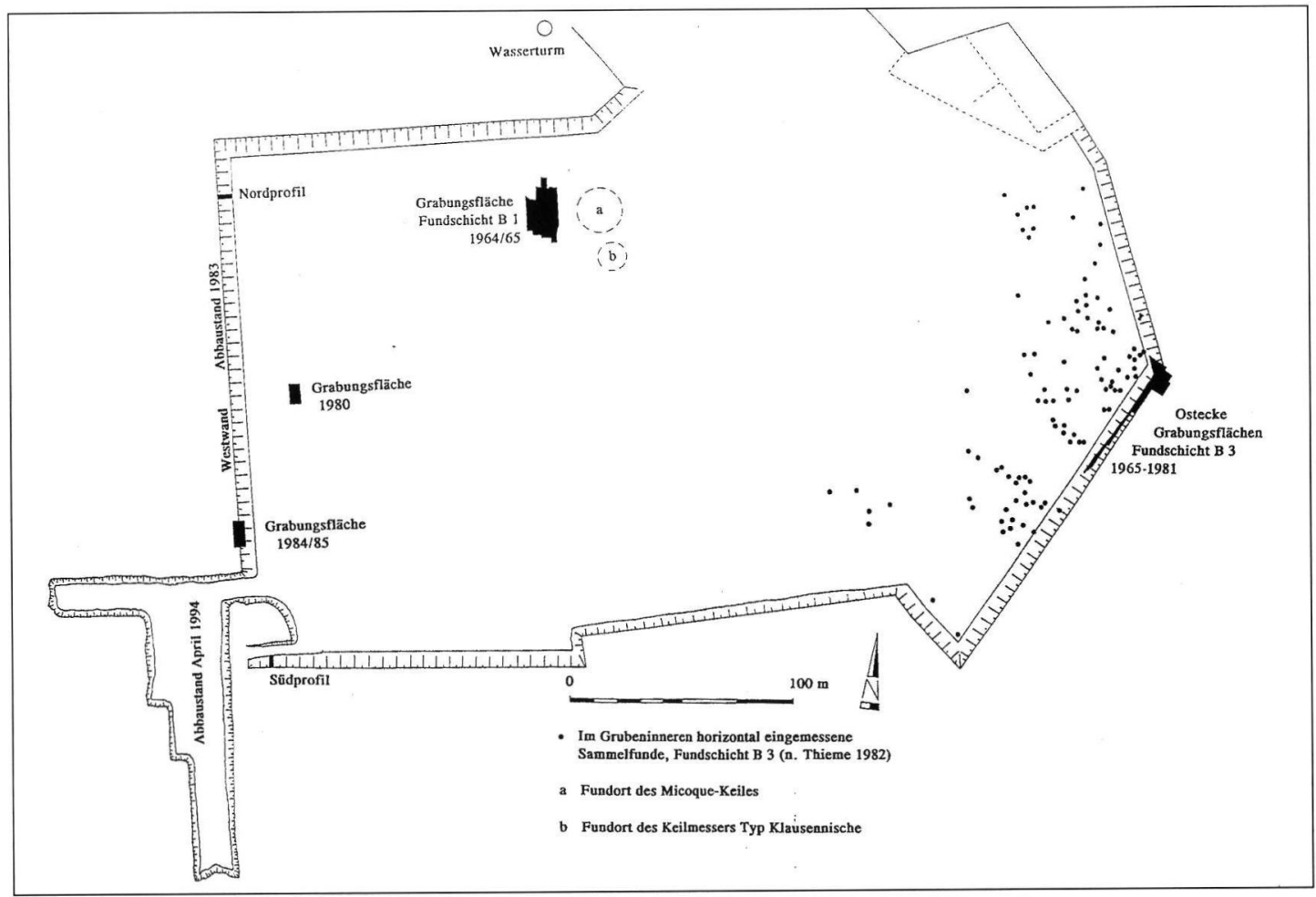

Abb. 3: Lageplan der Grube Dreesen.

Fig. 3: Situation of the Dreesen pit 
zeichnet. Für die jeweiligen Parabraunerden hat sich die Bezeichnung „Bt“ eingebürgert. Die Bt-Horizonte wurden vom Hangenden zum Liegenden hin durchnumeriert (s. Abb. 2 u. 4).

In den dreißiger Jahren entdeckte KaHRs (1951) bei geologischen Untersuchungen im Löß von Rheindahlen erstmals stratifizierbare Artefakte aus der Fundschicht B3 (Abb. 4). Seit dieser Zeit ist die Lößfolge von Rheindahlen oft beschrieben worden, zuletzt von Klostermann (1992) und SCHIRMER \& FELDMANN (1992).

Bereits 1949 fand eine Sondage durch NARR in der Fundschicht B3 im Löß des Saale-Komplexes statt (NARR 1951). Die ersten großflächigen Untersuchungen in Rheindahlen führte 1964/65 BOsINSKI in der Ostecke der Grube (Fundschicht B3) und in der Westwand-Fundschicht B1 durch (s. Abb. 3). Die B1-Fundschicht liegt im und auf dem Rocourt-Boden (2. Bt), der im Eem-Interglazial gebildet wurde. Die archäologischen Arbeiten in Rheindahlen setzte von 1973 bis 1981 Thieme fort. Neben den Fundschichten B1 und B3, die sich großflächig über fast den gesamten Grubenbereich und darüber hinaus erstrecken, wurden weitere acht paläolithische Fundhorizonte beschrieben (THIEME et al. 1981). Im Frühjahr 1984 entdeckte einer der Verfasser (J. THIsSEN) erneut eine Konzentration von Feuersteinartefakten in der Westwand-Fundschicht (B1) im Südwesten der Grube, die in den Jahren 1984/85 untersucht werden konnte (THISSEN 1986;1988).

\section{Das Westwandprofil der Ziegeleigrube Dreesen/Rheindahlen}

Die unterschiedlich alten Lößaufwehungen liegen Kies- und Sandschüttungen des Rheins aus der Jüngeren Hauptterrassen-Zeit auf. Die aufgeschlossene Schichtenfolge muß daher in der Zeit zwischen dem Cromer-Komplex und dem Holozän aufgeweht sein. Wie erwähnt, wird der gesamte Löß-Komplex durch vier Parabraunerden untergliedert. Das bedeutet, daß dort vier volle Warmzeiten dokumentiert sind. Die Parabraunerden wurden von BRUNNACKER (1973) zunächst rein deskriptiv von oben nach unten als 1. Bt bis 4. Bt bezeichnet (vgl. Abb. 2). Aufgrund seiner Fazies wurde der Löß zwischen 2. und 3. Bt als Fleckenlehm, jener unter dem 3. Bt als Staublehm bezeichnet. Diese Benennungen werden im vorliegenden Beitrag beibehalten. Im oberen Teil des Weichsellösses findet sich ein spätglazialer Verlagerungshorizont (verlagerte „Zone K“ bei BRUNNACKER 1973), der hier als Horizont K2 bezeichnet wird (vgl. Abb. 4). Im Südwesten der Grube fand sich unterhalb des rezenten Ah-Horizontes ein weiterer humoser Kolluvial-Horizont (K1).

Die erste stratigraphische Einstufung der Parabraunerden des Niederrheins und auch jener von Rhein- dahlen erfolgte bereits 1951 durch KaHRs. Diese Einstufung verfeinert BRUNNACKER (1966).

Unter Zuhilfenahme von Artefaktfunden wurde der 1. Bt, also der Tonverdichtungshorizont der obersten Parabraunerde, ins Holozän datiert. Der zweite Bt wurde dem Eem-Interglazial zugeordnet. Der dritte Bt muß folglich saalezeitlich oder älter sein. In den Jahren 1984 bis 1985 und später wurden von den Verfassern weitere Untersuchungen im Bereich der Westwand der Ziegeleigrube Dreesen durchgeführt.

\subsection{Geologische und bodenphysikalische Daten}

Die geologische Profilaufnahme zeigte, daß die $\mathrm{Pa}-$ rabraunerden des zweiten, dritten und vierten $\mathrm{Bt}$ nach Nordosten hin geneigt sind. Lediglich der erste Bt liegt mit seiner Basis parallel zur heutigen Geländeoberfläche. Er ist somit ungestört. Die Neigung der älteren Parabraunerden führte dazu, daß im äußersten Süden der Westwand der erste und der zweite Bt unmittelbar übereinander liegen. Im Nord-

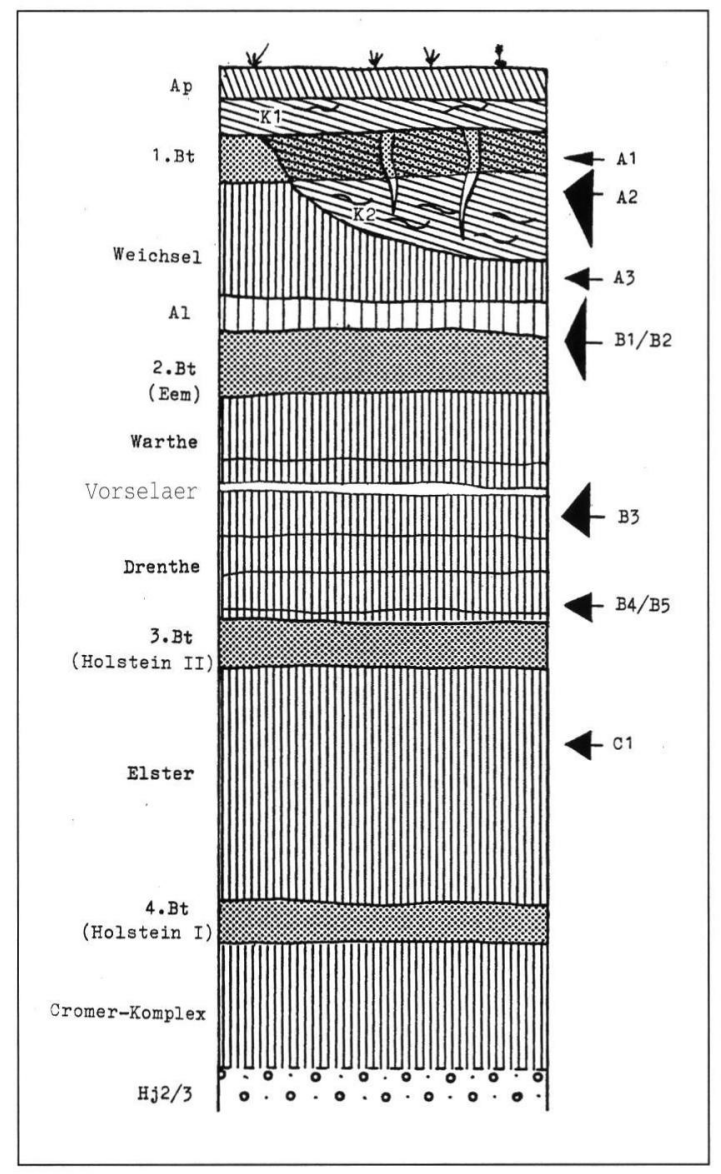

Abb. 4: Übersicht des Westwand-Profils.

Fig. 4: The profile of the western wall 


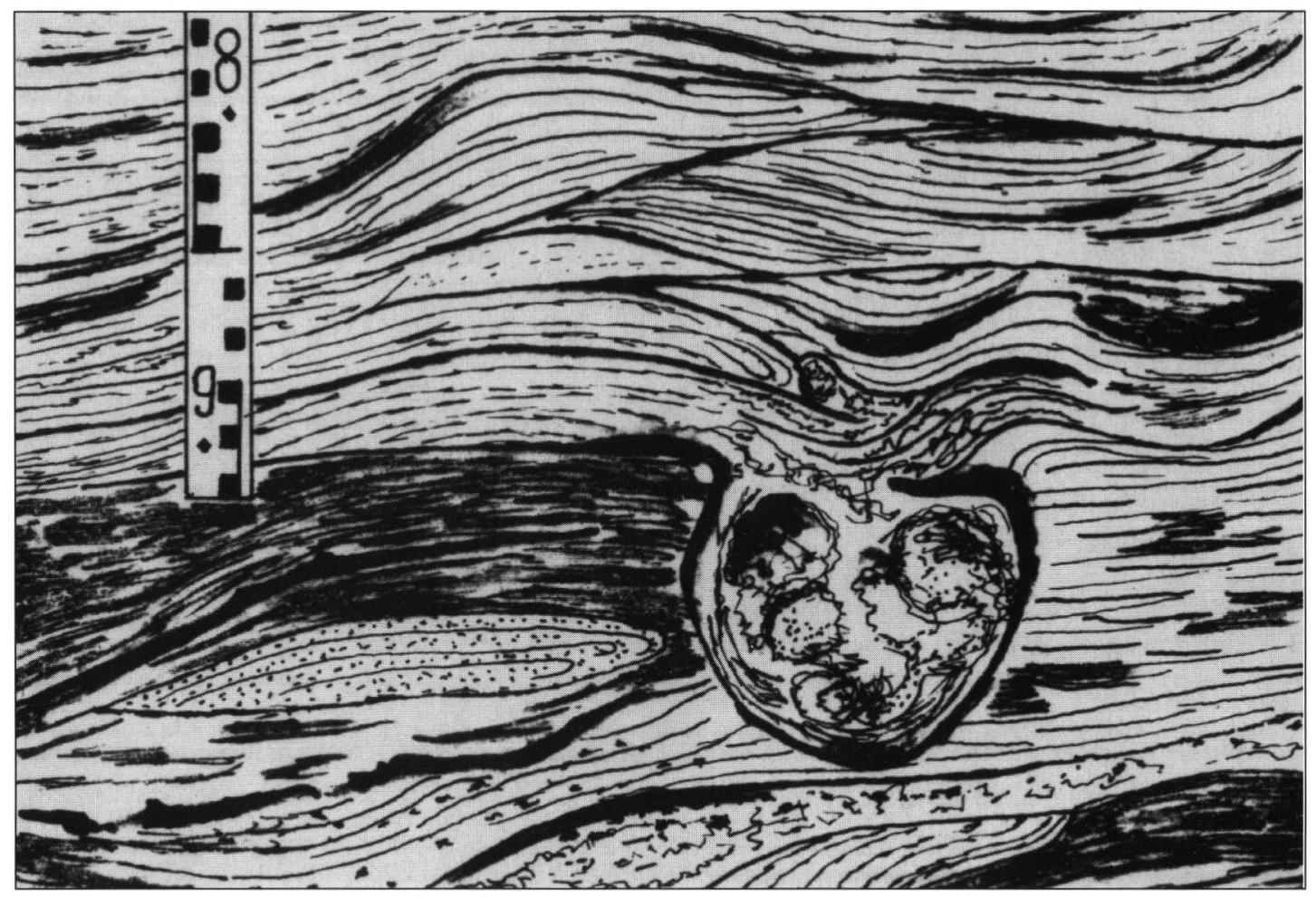

Abb. 5: Fließstrukturen im Nordprofil zwischen 1. und 2. Bt (Zeichnung: S. Hänschke)

Fig. 5: Flow structures in the northern profile between 1st and 2nd Bt-horizon.

abschnitt der Westwand wird deutlich erkennbar, daß der Löß zwischen dem ersten und zweiten Bt solifluktiv und kryoturbat verändert ist (s. Abb. $4 \mathrm{u}$.
5). Das bedeutet, daß darin vorgenommene Datierungen, welcher Art auch immer, keine stratigraphisch relevanten Daten liefern können.

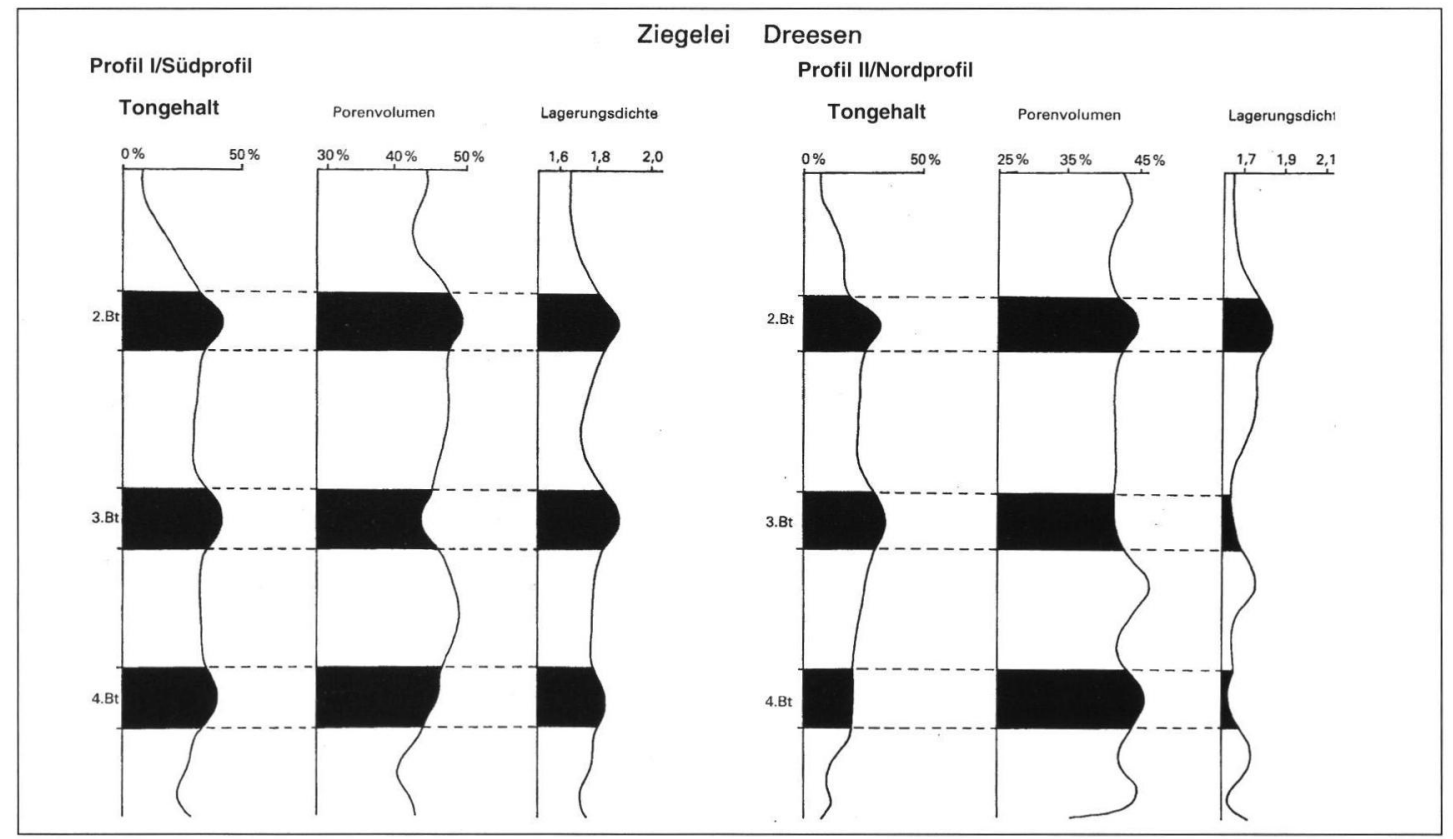


Der zweite Bt, der bereits in nördlicher Richtung eingekippt ist, besitzt im unmittelbaren Hangenden einen Al-Horizont, der auch als weißes Band bezeichnet wird. Das weiße Band ist sehr ausgeprägt entwickelt. Diese Tatsache spricht dafür, daß der zweite Bt während einer ausgeprägten Warmzeit entstanden sein muß. Die bodenphysikalischen Untersuchungen (ausgeführt von H. WICHTMANN, Geologisches Landesamt NRW) des zweiten Bt-Horizontes zeigen die höchsten Tongehalte des gesamten Westwandprofils (s. Abb. 6). Auch die Lagerungsdichte ist im zweiten Bt auffallend hoch. Identische Ergebnisse zeigen die Untersuchungen des zweiten Bt's im Nordprofil. Dort weisen die bodenphysikalischen Parameter im Hangenden des zweiten Bt deutlich darauf hin, daß die Schichten umgelagert wurden. Die Porenvolumina sind, ebenso wie die Lagerungsdichten, auffallend gering,

Auch der dritte Bt läßt sich mittels bodenphysikalischer Daten gut identifizieren. Hier ist der Tonanteil deutlich erhöht.

Höhere Lagerungsdichten treten beim dritten $\mathrm{Bt}$ im Südprofil erst unmittelbar im Liegenden des Bt auf. Dieser Horizont war offenbar nach seiner Entstehung intensiv wirkenden periglazialen Vorgängen ausgesetzt. Die Parabraunerde des dritten Bt lag also längere Zeit an der Erdoberfläche. Es ist daher mit großer Wahrscheinlichkeit am Top des dritten Bt ein Hiatus zu postulieren. Auch die übrigen Parabraunerden sind gekappt, fehlt doch mit Ausnahme des zweiten $\mathrm{Bt}$ der Al-Horizont.

Der vierte Bt läßt sich aufgrund bodenphysikalischer Daten an keiner Stelle im heutigen Westwandprofil mit Sicherheit nachweisen. Lediglich im Nordprofil (vgl. Abb. 6) sind die Porenvolumina im Bereich des vermuteten vierten $\mathrm{Bt}$ erhöht. Tonanteil und Lagerungsdichte geben jedoch keine Hinweise auf die Existenz dieses Bt im Westwandprofil. Das Niveau des. 4. Bt läßt sich jedoch mittels einer vorhandenen Verfärbung am Westwandprofil verfolgen (vgl. Abb. 6). Möglicherweise sind die typischen bodenphysikalischen Merkmale eines Bt durch spätere Überprägung aus bodenphysikalischer Sicht vollständig verloren gegangen. Weiter östlich war der vierte $\mathrm{Bt}$ jedoch mit Sicherheit vorhanden (Thieme et al. 1981: 41).

An der Basis des Nordprofils ist direkt über den hauptterrassenzeitlichen Kiesen und Sanden eine Schicht mit auffälligen bodenphysikalischen Parametern aufgeschlossen. Die Porenvolumina sind sehr gering (22,3 Vol. \%), die Lagerungsdichten extrem hoch. Es handelt sich bei dieser Schicht mit großer Wahrscheinlichkeit um Hochflutablagerungen aus der Jüngeren Hauptterrassen-Zeit.

\subsection{Schwermineralgesellschaften und Floren}

Die Schwermineralspektren werden aufgrund der Feinkörnigkeit des Lösses von stabilen Schwermine- ralen dominiert. Im 1. Bt ist Laacher-See-Tephra nachweisbar (THIEME et al. 1981). Im Gesamtspektrum (Untersuchung: U. Wefels, Geologisches Landesamt NRW) herrschen Zirkon und Rutil vor. Auch Epidot kommt in größeren Prozentanteilen vor. Die Bt-Horizonte der Parabraunerden werden besonders deutlich vom Granat nachgezeichnet. Sowohl im zweiten als auch im dritten Bt des Nord- und des Südprofils gehen die Granatanteile innerhalb der BtHorizonte deutlich zurück. Die Zirkon- und Rutilgehalte sind im zweiten $\mathrm{Bt}$ des Nordprofils und im dritten Bt des Südprofils deutlich erhöht. Bei den vorliegenden Untersuchungen spielen jedoch offenbar Korngrößeneffekte eine entscheidende Rolle. In beiden Profilen zeigen Zirkon und Rutil im Liegenden des dritten Bt einen einheitlichen Kurvenverlauf. Die Prozentanteile steigen in beiden Profilen von oben nach unten hin an. In gleicher Weise verhält sich der Granat. Die Epidot-Werte sinken dagegen im Liegenden des dritten $\mathrm{Bt}$ deutlich ab, verlaufen aber ebenfalls etwa parallel. Die Schichtenfolge im Liegenden des dritten $\mathrm{Bt}$ dürfte daher aufgrund der Schwermineralspektren stratigraphisch in das gleiche Niveau einzuordnen sein. Klinopyroxen und braune Hornblende treten allenfalls mit 0,5 oder $1 \%$ auf und lassen somit keine genaue stratigraphische Einstufung zu. Eine im oberen Teil der Fundschicht B 3 (vgl. Tab. 1) angeblich vorhandene Tephra-Lage wurde schwermineralanalytisch untersucht. Im Schwermineralspektrum kommen jedoch weder Klinopyroxen noch braune Hornblende noch Titanit vor. Zirkon, Turmalin und Epidot machen $96,5 \%$ des Spektrum aus. Bei der in Rede stehenden Schicht handelt es sich daher mit Sicherheit nicht um eine Tephra-Lage.

Wie nicht anders zu erwarten, erlauben auch die pollenanalytischen Daten in der Regel keine exakte stratigraphische Aussage. In den Proben des Südprofils (beide Profile wurden von R. STRITZKe, Geologisches Landesamt NRW, untersucht) fanden sich bis $1,8 \mathrm{~m}$ Tiefe Pollen bzw. Sporen von Corylus, Nyssa, Betula, Pinus, Picea, Plantago lanceolatum, Artemisia, Lycopodium, Pocacea, Liguliflorae sowie Chenopodiaceae. Auch im Nordprofil sind die Ergebnisse ausgesprochen dürftig. Dort fanden sich in $1 \mathrm{~m}$ Tiefe folgende Pollen: Pinus, Corylus, Cerealia, Plantago lanceolatum, Chenopodiaceae, Caryophyllaceae, Cichoricae, Centaurea, Rosaceae, Ranunculaceae, Pocaceae und Ericaceae. Sphagnum, Lycopodium, Osmunda, Anthoceros und Filicines waren nur durch wenige Sporen vertreten. Wichtig ist jedoch, daß sich im Nordprofil im humosen Kolluvium K1 auch kulturanzeigende Pollen fanden. Dieser Teil des Profiles ist daher mit hoher Wahrscheinlichkeit dem Holozän zuzuordnen.

Bei der Grabung 1984/85 geborgene Holzkohlenstückchen aus der Fundschicht B 1 konnten für eine 
Holzartbestimmung herangezogen werden. Acht der neun Holzkohlen stammen aus der „Übergangszone $\mathrm{B} / \mathrm{C}$ ". Dort fanden sich auch die meisten Artefakte. Einer der Funde lag im sogenannten hellen Band. Die Bestimmung der Holzkohlen ergab $3 \mathrm{mal}$ Ahorn, 2mal Birke, 2mal Hainbuche, 1mal Eiche und 1mal Seidelbast (THISSEN 1988: 47 f). Die bestimmten Hölzer belegen den warmzeitlichen Charakter dieses Schichtenabschnitts.

\subsection{Die Artefaktinventare}

\subsubsection{Fundschicht A 1}

Aus dem jüngsten paläolithischen Horizont, der Fundschicht A1, stammen zwei unpatinierte Feuersteinartefakte, die ca. $60 \mathrm{~cm}$ unterhalb der Geländeoberfläche im 1. Bt gefunden wurden (THIEmE et al. 1981, 47 f). Es handelt sich dabei um eine geknickte Rückenspitze und um einen partiell retuschierten Abschlag (Taf. 1, 1 u. 2). Die Rückenspitze gehört formenkundlich in ein spätes Jungpaläolithikum (Magdalénien) oder Spätpaläolithikum (Federmessergruppen); dies umfaßt den Zeitraum von etwa 13000 - 10000 BC (Thissen 1989; 1994). Demnach ist die von SChIrmer \& Feldmann (1992) als EemBoden angesprochene Parabraunerde zweifelsfrei als postglaziale Bildung (1. $\mathrm{Bt}$ ) anzusehen.

\subsubsection{Fundschichten A2 und A 3}

Aus dem Löß der letzten Kaltzeit (Weichsel), Fundschicht A3, stammt ein Faustkeil aus Tertiärquarzit (Taf. 1, 3), der typologisch als MTA-Keil (Moustérien de tradition acheuléenne) angesprochen wurde (BoSINSKI 1983: 91; THIEME et al. 1981: 48 ff). Die diskutierte Provenienz des Rohmaterials (Liedberg(?)Quarzit) ist fraglich, da ein solcher Quarzit häufig isoliert in den Flußschottern des Niederrheingebietes vorkommt (THISsen 1993). Das MTA datiert in die erste Hälfte der letzten Kaltzeit, der Lößlehm unterhalb des postglazialen Bodens ist also im WeichselGlazial abgelagert bzw. umgelagert worden. Zahlreiche weitere Artefakte aus dem Weichsel-Glazial (Fundschicht A 2 und A 3) - darunter sind Faustkeile, Schaber, Levalloisabschläge, retuschierte Klingen und ein Keilmesser - haben ein allgemein spätmittelpaläolithisches Gepräge (BOSINSKI 1971).

\subsubsection{Fundschicht B 1/B 2}

Die von THIEME beschriebenen Fundhorizonte B1 und B2 (ThIEME 1983 a: 57 ff) gehören nach neuesten Erkenntnissen einer gemeinsamen Fundschicht an (SCHMiTz \& Thissen 1994). Während der Arbeiten von BOsINSKI in der Ostecke (Fundschicht B 3) wurde im Oktober 1964 die Westwand-Fundschicht (B1) entdeckt. Bosinski beschrieb die Stratigraphie so: „Unter einem mehrfach gegliederten gelben Lößlehm folgt eine schwach humose Strate (A), darunter ein durchschnittlich $15 \mathrm{~cm}$ mächtiges, helles, tonarmes, in feinen Streifen horizontal geschichtetes Band (B) und dann ein $50 \mathrm{~cm}$ starker rotbrauner Lößlehm (C). Zwischen der hellen Schicht B und der rotbraunen Schicht $C$ liegt eine breitere Übergangszone (B/C)" (Bosinski 1966: 320). Diese Schichtenabfolge konnte bei den Untersuchungen 1984/85 in der Westwand-Fundschicht in wesentlichen Teilen bestätigt werden (THISseN 1986). Lediglich die humose Zone A wurde nicht erkannt, da im Südwesten der Grube der weichselzeitliche Lößlehm durch tiefgreifende Erosionsvorgänge (K2) bis auf das helle Band hinab abgetragen wurde. Bei den Ausgrabungen 1984/85 stellte sich heraus, daß die Funddichte nach unten zwar deutlich abnahm, doch fanden sich bis zum letzten Planum in der Mitte des Rocourt-Bodens vereinzelt Artefakte, die eindeutig zur Fundschicht B1 gehören (THISsen 1988: 65 ff).

Die vertikale Streuung der Artefakte betrug 60 Zentimeter. Eine solche vertikale Dynamik von Steinartefakten kann durch „Auffrieren“ erfolgen, da von der Oberfläche her eindringender Frost durch Steine aufgrund ihrer guten Temperaturleitfähigkeit schnell nach unten weitergleitet wird. Dies führt zu einem relativ frühen Gefrieren des im Sediment enthaltenen Wassers unterhalb der Artefakte. Da das Wasser beim Übergang in den festen Aggregatzustand eine Volumenzunahme von knapp $10 \%$ erfährt, werden die Steine durch abwechselndes Gefrieren und Auftauen jeweils um einen geringen Betrag angehoben; geschieht dies über einen längeren Zeitraum, kann es zu einer vertikalen Verlagerung von einigen Dezimetern kommen. Andererseits können auch Bioturbationsprozesse während oder nach dem Besiedlungsvorgang dafür gesorgt haben, daß Steingeräte einige Dezimeter unter den eigentlichen Laufhorizont geraten sind.

Die ergrabenen Steingeräte der Fundschicht B 1 sind im allgemeinen wenig charakteristisch und formenkundlich kaum aussagekräftig, typische mittelpaläolithische Formen fehlten bisher. Unter den $1484 \mathrm{Ar}-$ tefakten der Grabung 1964/65 (Taf. 1, 4 - 8) fanden sich 30 Werkzeuge und 25 partiell retuschierte Formen, häufig an gestreckten Abschlägen oder Klingen (Bosinski 1966: 320). Die Bearbeitung beschränkte sich auf die Kanten, Flächenretusche kommt bei diesen Stücken nicht vor. In der gleichen Art wurden auch die wenigen Schaber hergestellt. Die Produktion der Grundformen erfolgte wenig systematisch, die Kerne wurden von allen sich anbietenden Schlagflächen her abgebaut. Dabei entstanden eher zufällig einige wenige Klingen. Typische Klingenkerne fehlen sowohl im Material der Grabung 1964/65 als auch in dem der Grabung 1984/85. 
Für die Anwendung einer planmäßigen Klingentechnologie, wie sie für die mittelpaläolithischen Stationen Seclin, Rocourt oder Riencourt-les-Bapaume beschrieben wird (TuFfreau et al. 1985; CAHEN \& Haesaerts 1984; Tuffreau \& Almeloot-Van Der HeiJDEN 1990), gibt es aus der Fundschicht B 1 in Rheindahlen bisher keine Anhaltspunkte.

Von den Artefakten der Grabung 1964/65 konnte THIEME durch umfangreiche Zusammensetzarbeiten $48,5 \%$ aller Stücke wieder anpassen, was einem Gewichtsanteil von $88 \%$ des gesamten Inventars entspricht (Thieme 1983 a: 60). Bei diesen Untersuchungen gelang es THieme auch, unterschiedliche Arbeitsbereiche innerhalb des Siedlungsareals (280 $\mathrm{m}^{2}$ ) herauszuarbeiten (THIEME 1983 b: $362 \mathrm{ff}$ ).

Unter den 277 Artefakten der Grabung 1984/85 (Taf. 1, 9 - 13) fanden sich nur fünf retuschierte Formen, darunter ein kleines Klingenbruchstück mit einer feinen sägezahnartigen Retusche (Taf. 1, 9). Von diesen konnten $18,05 \%$ aller Stücke wieder zusammengesetzt werden; berücksichtigt man die Absplisse $<10 \mathrm{~mm}$ nicht,

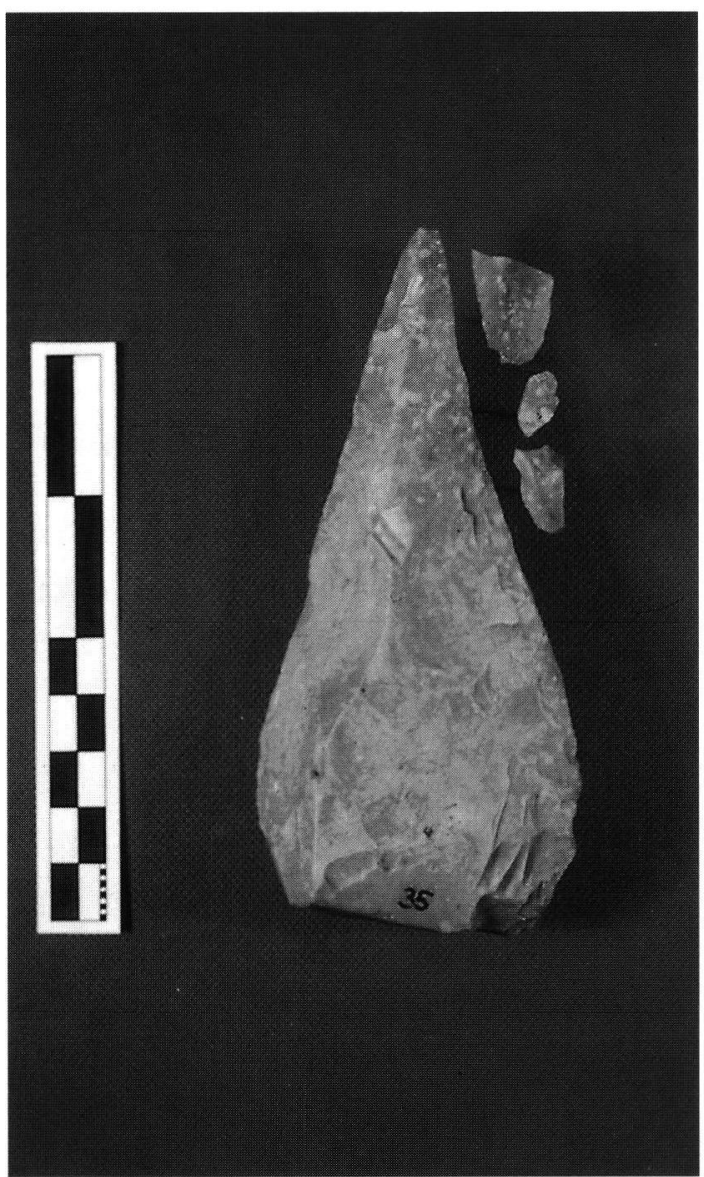

Abb. 7: Micoque-Keil, Fundschicht B 1/B 2 mit zugehörigen Abschlägen

Fig. 7: Micogue artefact, finding layer B1/B2 with combined cut off parts. so betrug die Zusammensetzrate 33,8 \% (THISSEN 1988 : 92). Insgesamt belegen die Untersuchungsergebnisse für die Fundschicht B 1, daß eine horizontale Verlagerung des Siedlungsplatzes kaum oder nur in sehr geringem Umfang stattgefunden haben kann.

Aus der Mitte des Rocourt-Bodens stammt ein Faustkeil mit wechselseitig-gleichgerichtet bearbeiteten Kanten, einer leicht asymmetrisch ausgezogenen Spitzenpartie und verdickter Basis (Taf. 2, 1). Aufgrund seiner stratigraphischen Position im Eem-Boden, dessen Ausgangsgestein der Löß der vorletzten Kaltzeit (Warthe) ist, stellte Thizme diesen Faustkeil und ein weiteres bifaziales Gerät in ein „Jungacheuléen nordfranzösischer Ausprägung" (THIEmE 1983 a: 124 f). Diese beiden Artefakte ordnete er einer Fundschicht „B2“ zu. Formenkundlich ist der Faustkeil zweifelsfrei als Micoque-Keil anzusprechen, und so beschreibt auch Bosinski das Artefakt als den ,... wohl schönste(n) Micoquekeil des Arbeitsgebietes ..." (Bosinski 1967: 49). Die stratigraphische Position in der Mitte des 2. Bt entspricht der Unterkante der Fundschicht B 1 der Grabung 1984/85. Weiterhin wurde der Faustkeil unmittelbar vor der Grabungsfläche 1964/65 (s. Abb. 3), nur wenige Meter von dieser entfernt, gefunden (mündl. Mitt. A. Mennen). Dabei, so Mennen, konnte die stratigraphische Position des Micoquekeiles innerhalb des Eem-Bodens nachträglich nicht mehr ganz genau geklärt werden, doch ist die Herkunft „2. Bt" gesichert. Das Stück besteht aus einem grünlich-grauen Feuerstein mit hellen Einsprenglingen, der stellenweise schwach milchig-weiß patiniert ist; andere Partien wirken unpatiniert. Beim Vergleich des Faustkeils mit den Artefakten der Grabung 1964/65 fand sich bei einer kürzlich vorgenommenen Durchsicht des Materials im Rheinischen Landesmuseum Bonn ein Abschlag (lfd. Nr. 282), der der Rohmaterialeinheit des Faustkeils mit hoher Wahrscheinlichkeit angehört und unter den 1964/65er Rohmaterialvarietäten als „Einzelstück“ auffiel. THIEME bemerkte zu diesen Einzelstücken: „..., so verbleibt immer noch eine Anzahl von ca. 50 Rohmaterialeinheiten, die bis auf eine oder nur wenige Grundformen den Siedlungsplatz nicht erreicht haben ... Es ist anzunehmen, daß der Hauptteil dieser Knollen weitgehend auf Siedlungsstellen aufgebraucht worden ist, die dem „Rheindahlener" Siedlungsvorgang vorausgingen oder sogar gleichzeitig mit diesem genutzt worden sind" (ThIEME 1983: 100). Bei den Untersuchungen 1984/85 konnte gezeigt werden, daß sich schon in unmittelbarer Nähe der Siedlungsaktivitäten der 1964/65er-Fläche, etwa 200 Meter südwestlich, weitere Zonen spezifischer Aktivitäten, unter anderem mit Feuersteinverarbeitung, befanden. Unter den 277 Artefakten fanden sich ebenfalls Einzelstücke, die keiner Rohmaterialeinheit der Grabung 1984/85 zugeordnet werden konnten. Besonders zu 
nennen sind hier drei kleine Abschläge, die mit sehr hoher Wahrscheinlichkeit Retuschierabfälle des „B 2"-Faustkeils sind (s. Abb. 7). Von der Art des Rohmaterials her sind diese mit dem Micoquekeil identisch; der Versuch einer Anpassung an den Faustkeil ist bisher nicht geglückt.

Weiterhin entdeckte A. MENNEN in der Grubeninnenfläche zahlreiche Steinartefakte, die beim fortschreitenden Lößabbau aus der Wand gefallen sind. Darunter sind ein Keilmesser Typ Klausennische (Taf. 2/2), das MenNen unmittelbar südöstlich der Grabungsfläche 1964/65 auflas, und ein Bocksteinmesser (Taf. 2, 3) aus einem Maasei, das 1971 südwestlich der Grabung 1964/65 (nicht mehr genau zu lokalisieren) gefunden wurde. Beides sind typische Formen des Micoquien und gehören vermutlich ebenfalls in die Fundschicht B1.

Ebenfalls in diesem Zusammenhang ist eine dünne Fundstreuung in der B1-Fundschicht zu nennen, die THIEme 1980 etwa 150 Meter westlich der von Bosinski 1964/65 ausgegrabenen Fläche untersuchen konnte (Thieme 1980: $57 \mathrm{ff}$ ). Auf einer Fläche von $50 \mathrm{~m}^{2} \mathrm{fan}-$ den sich etwa 50 Artefakte. Die Siedlungsaktivitäten in der Westwand-Fundschicht B 1 erstreckten sich insgesamt über ein Areal von mindestens $10000 \mathrm{~m}^{2}$. Aufgrund der stratigraphischen Position der Fundschicht B 1, der archäologischen Ansprache des Inventars und der geobotanischen Untersuchungen (s. o.) kann der zeitliche Rahmen für den Besiedlungsvorgang auf das Eem-Interglazial und die frühweichselzeitlichen Interstadiale beschränkt werden. Der „B 2“-Faustkeil gehört aufgrund der weiter oben beschriebenen Beobachtungen höchstwahrscheinlich zur Fundschicht B 1. Da die Fundschicht B 1/B2 mit einem eem- oder frühweichselzeitlichen Alter in den postsaalezeitlichen Komplex gehört, müßte diese konsequenterweise mit „A4" bezeichnet werden, doch wird aufgrund einer terminologischen Einheitlichkeit die Bezeichnung „B1/B2“ beibehalten. Durch die Zugehörigkeit des Micoquekeiles zu der Fundschicht B1/B2 wird diese formenkundlich ansprechbar. Somit kann die geochronologische Einstufung des bisherigen (und tatsächlichen) 2. Bt von Rheindahlen in das Eem-Interglazial archäologisch bestätigt werden.

\subsubsection{Fundschicht B 3}

Die Artefakte der Fundschicht B3 (Ostecken-Komplex) stammen aus dem Löß unterhalb des Eem-Bodens (Taf. 3, 1 - 10); es liegen Spitzen mit konvexen Kanten, einfache Schaber, wenige Breitschaber, Doppel- und Spitzschaber vor. Weiterhin findet man retuschierte Abschläge, Klingen, kielartige Stücke, Quinson-Spitzen und das Bruchstück einer Limace (THISSEN 1990: 63 ff). Unter den Nicht-Flintgeräten sind hackenartige Werkzeuge, Chopper und Chop- ping tools aus Quarz und Quarzit vertreten, ebenso Reib- oder Schleifplatten aus quarzitischem Sandstein (THIEme et al. 1981: 57 ff). Bei diesem Inventar aus der Mitte des Fleckenlehms handelt es sich um ein Charentien Typ Ferrassie (Bosinski 1993: 39 f). Dieses geradezu klassische Geräte-Inventar aus der Zeit der Neandertaler soll nach den Ergebnissen von SChirmer \& Feldmann (1992) in die Kaltzeit vor den Saale-Komplex datieren, was bedeuten würde, daß man dieses Inventar dem Homo erectus zuschreibt und in ein Altpaläolithikum stellt!

Bei der geochronologischen Einstufung des Lösses sind durch THIEME aus der Fundschicht B 3 geborgene Holzkohlen von Bedeutung. Von den 27 schlecht erhaltenen Proben wurden bestimmt: 2 (nahe) Fraxinus, 2 (nahe) Salix, 1 Alnus oder Corylus, 1 Quercus (?), 1 Betula und 1 Nadelholz (?) (Thieme 1983 a: 26). Zahlreiche weitere Proben waren nicht bestimmbar, doch deutet die hohe Zahl von Laubhölzern auf gemäßigte klimatische Bedingungen hin. Obwohl in diesem Bereich des saalezeitlichen Lösses keine fossile Bodenbildung erhalten ist, muß die Besiedlung, bei der die Artefakte der Fundschicht B3 hinterlassen wurden, in zeitlicher Nähe einer warmgemäßigten Klimaphase stattgefunden haben (SChMiTz \& THISsen 1994). Im oberen Bereich der bis zu $50 \mathrm{~cm}$ in der Vertikalen streuenden Fundschicht B 3 durchzieht ein ca. $6 \mathrm{~cm}$ mächtiges helles Bändchen den Löß des Saale-Komplexes. Dieser Naßboden (THIEME 1983 a: 23) hält auf der gesamten Länge der Westwand durch. Nach THIEME ist es nicht auszuschließen, daß sich hier eine erhebliche Erosionsdiskordanz innerhalb des Fleckenlehms andeutet. Möglicherweise liegt ein reliktisch erhaltener Bodenrest vor (SCHMitz \& THISSEN 1994).

\subsubsection{Fundschicht B4/B5}

Die 1969 von MENnEN entdeckte Fundschicht B5 liegt an der Basis des saalezeitlichen Lösses (BosinsKI \& Brunnacker 1973). Die zwölf bisher in situ geborgenen Einzelfunde, die sich über die gesamte Westwand erstreckten, sind unpatiniert. Neben Artefakten aus Maasschotterfeuersteinen liegen zwei angeschlagene Quarzgerölle und ein Chopper aus Quarzit vor. Bei den Flintartefakten handelt es sich um zwei präparierte Kerne zur Produktion von Abschlägen (Taf. 4,1) und klingenartigen Formen (Taf. 4,2); daneben findet man entsprechende Levalloisabschläge (Taf. 4,4) und eine regelmäßige Levalloisklinge mit alternierend retuschierter Spitzenpartie (Taf. 4,3). Zwar fehlen bisher Faustkeile, doch läßt sich dieses Inventar aufgrund seiner stratigraphischen Position und anhand des vorliegenden Formenspektrums am ehesten in das Jungacheuléen einordnen (SchMiTz \& THISSEN 1994). ThiEME möchte das Inventar einer älteren Phase des Acheuléen (Mit- 
telacheuléen) zuweisen (ThiEme et al. 1981: 62). Gegen diesen Ansatz spricht unseres Erachtens sowohl die geochronologische Stellung der Fundschicht B5 als auch die Anwendung einer guten Levalloistechnik, wie sie in dieser Ausprägung für den Homo erectus bisher nicht belegt ist. Drei etwa $20 \mathrm{~cm}$ oberhalb der Fundschicht B5 in situ geborgene Abschläge weist THIEME einer jüngeren Fundschicht „B4“ zu (Thieme et al. 1981: 62). Die unpatinierten und mit „hartem“ Schlag gewonnenen Feuersteinabschläge weisen glatte (2mal) und facettierte (1mal) Schlagflächenreste auf; die Zuordnung zu einer Formengruppe ist nicht möglich: Ihre stratigraphische Nähe zur Fundschicht B 5 läßt jedoch vermuten, daß sie ebenfalls zu dem oben beschriebenen Inventar gehören. Bei den über viele Jahre durchgeführten planmäßigen Untersuchungen in den Fundschichten B 1/B 2 und B 3 konnte gezeigt werden, daß die vertikale Artefaktstreuung der Fundschicht B 3 regelhaft etwa $50 \mathrm{~cm}$ betrug (THIEme 1983 a: $126 \mathrm{ff}$ ); in der Fundschicht B 1/B 2 waren dies bis zu $60 \mathrm{~cm}$ (SchmiTz \& THISSEN 1994). Eine gewisse vertikale Dynamik ist auch für die Fundhorizonte B 4/B 5 anzunehmen, so daß eine Differenzierung in zwei Fundschichten bei der geringen Artefaktzahl nicht gerechtfertigt erscheint.

Geochronologisch paßt ein solches Inventar mit Anwendung der Levalloistechnik und einem wenig intensiven Ausnutzungsrad der Kerne nach bisherigen Erkenntnissen gut in die erste Hälfte des Saale-Komplexes (Drenthe?).

\subsubsection{Fundschicht C1}

Die Fundschicht C1 wurde 1977 im Staublehm 20 cm unterhalb des 3. Bt entdeckt und auf einer Fläche von 5,4 $\mathrm{m}^{2}$ untersucht (Thieme 1977, 1981: 65). Der Schicht C 1 entstammen drei Quarzartefakte (Taf. 4, 5 - 7); neben einem angeschlagenen Quarzgeröll liegen zwei Kortexabschläge mit partieller Kantenretusche und Gebrauchsaussplitterungen vor. Von Bedeutung ist, daß diese Stücke ein geschlossenes, in situ geborgenes Inventar darstellen. Die chronostratigraphische Position unterhalb des 3. Bt (Holstein?) macht die Zugehörigkeit zu einem „Altpaläolithikum" wahrscheinlich. Mit dem C 1-Inventar liegen die ältesten in situ geborgenen Relikte aus der Zeit des Homo erectus im Niederrheingebiet vor. Formenkundlich steht es dem Inventar von Vértesszöllös, einem ungarischen Travertinfundplatz, nahe, der auf ein "intra-mindelzeitliches" Alter datiert (VerTes 1965). Es ist daher dem Elster-Komplex zuzurechnen.

\section{Stratigraphische Einstufung der Parabraunerden}

Die in dieser Arbeit vorgestellten Untersuchungsergebnisse erlauben eine stratigraphische Zuordnung der im Westwandprofil sicher nachweisbaren BtHorizonte eins bis drei. Als gesichert muß angenommen werden, daß die oberste Parabraunerde ins Holozän zu stellen ist. Im Südprofil wurden in $1 \mathrm{~m}$ Tiefe mittels Pollenanalyse Kulturanzeiger nachgewiesen, so daß der oberste Bt nur holozänzeitliches Alter haben kann.

Der zweite Bt-Horizont läßt sich auch aufgrund der bodenphysikalischen Ergebnisse über die gesamte Westwand hinweg verfolgen, so daß die Analysenergebnisse aus dem Nord- und Südprofil miteinander verglichen werden können. So zeigen auch die schwermineralanalytischen Daten, daß der zweite Bt im Süd- und Nordprofil korrekt verknüpft wurde. Die Funde von Micoque-Geräten im und über dem zweiten Bt stützen die Einstufung in die EemWarmzeit. Eine Zuordnung des zweiten Bt in den Saale-Komplex oder noch ältere Schichten (SchIRMER \& FELDMANN 1992) ist aufgrund der hier vorgestellten Daten eher unwahrscheinlich.

Die in der Mitte des Fleckenlehms in der Fundschicht B 3 angetroffenen Holzkohlen belegen für diesen Abschnitt der Schichtenfolge eine Warmzeit. Deren stratigraphische Zuordnung wird durch Artefakte aus der Fundschicht B 3 sowie B 4/B 5 möglich. Das Fundinventar aus der Mitte des Fleckenlehms ist ein Charentien Typ Ferrassie. Derartige Funde sind nach bisherigen Erkenntnissen in den Saale-Komplex oder das Weichsel-Glazial zu stellen. Damit wird eine Zuordnung der Holzkohlenschicht zu einer saalezeitlichen Wärmeschwankung wahrscheinlich. Daß es tatsächlich zwischen Drentheund Warthe-Stadium eine Warmzeit gegeben hat, machen Untersuchungen am nördlichen Niederrhein wahrscheinlich (KLostermann \& Rehagen \& WeFELS 1988). Eine Zuordnung des Fleckenlehms in die Zeit vor dem Saale-Komplex (SCHIRMer \& FelDMANN 1992) würde eine Rückdatierung des Artefaktinventars in das Altpaläolithikum erforderlich machen. Für eine solche Altersstellung dieser Artefakte gibt es jedoch bisher keinen Hinweis. Für die stratigraphische Zuordnung des dritten Bt geben ebenfalls die Artefakte wichtige Hinweise. Nach den bisherigen Kenntnissen ist das Artefaktinventar der Fundschicht B4/B5 in die erste Hälfte des SaaleKomplexes zu stellen. Daraus folgt, daß der dritte Bt mit großer Wahrscheinlichkeit in das Holstein-Interglazial gestellt werden muß. Das bedeutet, daß die von Klostermann (1992) vorgenommene Zuordnung des dritten Bt in die "Treene-Warmzeit" nicht mehr haltbar ist. Die Einstufung des dritten Bt in das Holstein-Interglazial wird durch die Artefakte der Fundschicht C1 untermauert. Den dort gefundenen Artefakten wurde an anderen Orten ein ,intra-mindelzeitliches" Alter zugewiesen. Das bedeutet, daß die Funde vermutlich älter als das Holstein-Interglazial sind. 
Tab. 1: Stratisgraphische Stellung des Lößprofils von Rheindahlen Table 1: Stratigraphic position of the loess profile Rheindahlen.

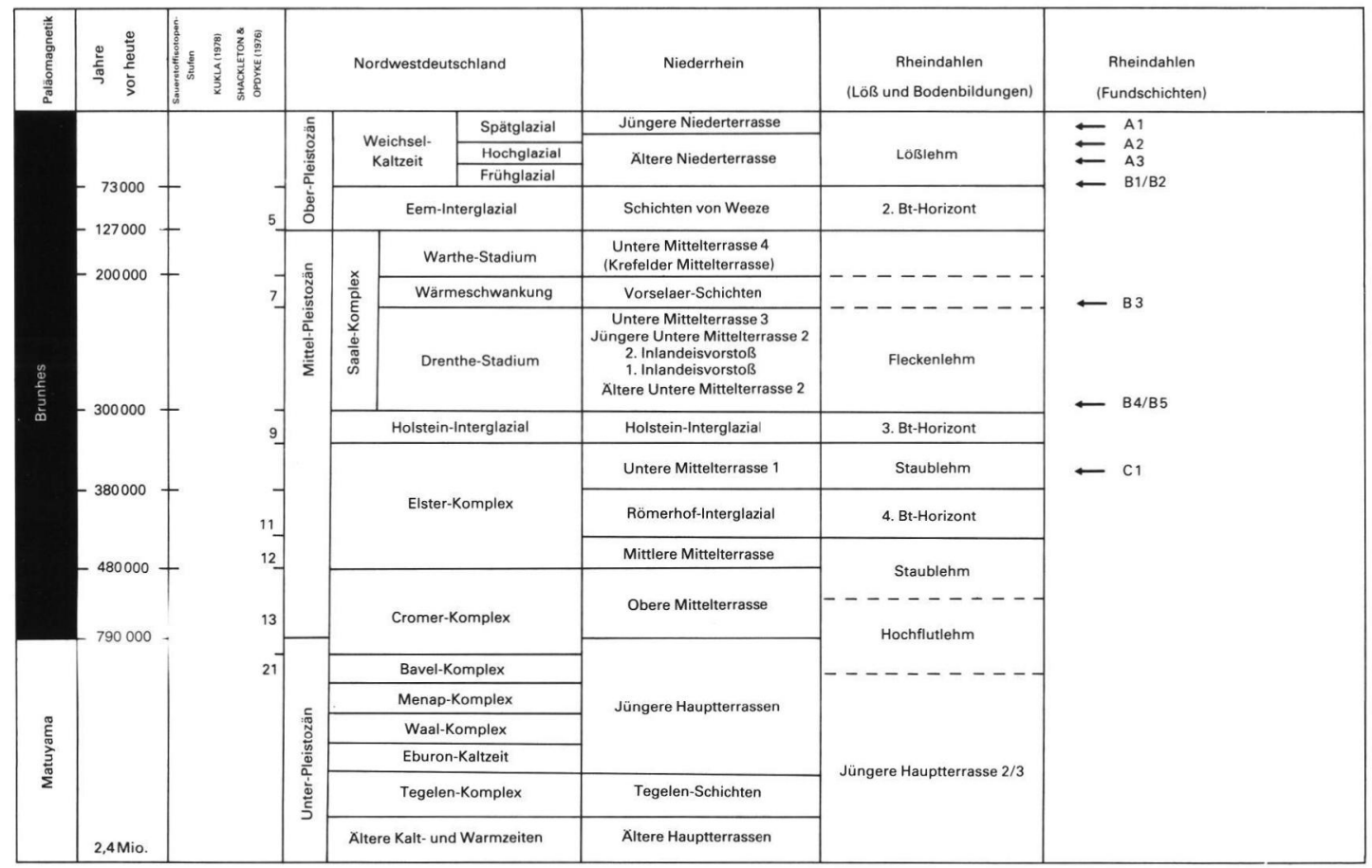

Der vierte Bt keilt jedoch offenbar nach Westen hin aus, so daß er im derzeitigen Westwandprofil nicht mehr nachweisbar ist. Dieser vierte Bt läßt sich unter großem Vorbehalt mit dem Römerhof-Interglazial verknüpfen (Klostermann 1995). Es kann jedoch nicht ausgeschlossen werden, daß hier eine noch ältere Warmzeit überliefert ist.

\section{Versuch einer chronostratigraphischen Einordnung}

Der Versuch einer chronostratigraphischen Korrelation gestaltet sich außerordentlich schwierig, da Thermolumineszenz-Datierungen offenbar zur Zeit noch keine hinreichend genauen Daten liefern. Als gesichert darf jedoch gelten, daß der zweite Bt, der der Eem-Warmzeit zugeordnet wird, mit der Sauerstoffisotopenstufe 5 e gleichgesetzt werden kann (s. Tab. 1). Die Entstehungszeit kann daher auf 127.000 bis etwa 110.000 Jahre BP eingegrenzt werden. Bei sämtlichen älteren Bt-Horizonten ist die chronostratigraphische Zuordnung nur mit Vorbehalt möglich. Da die Untersuchungen am Niederrhein gezeigt haben, daß es eine Intra-Saale-Warmzeit gegeben haben muß, ist es naheliegend, die Schicht mit Holzkohlenresten im Fleckenlehm mit der einer spätsaalezeitlichen Wärmeschwankung gleichzusetzen. Sie sollte in diesem Fall der Sauerstoffisotopenstufe 7 der Tiefseegliederung entsprechen. Das absolute Alter dieser Schicht dürfte daher bei etwa 200.000 Jahren BP liegen. Das Holstein-Interglazial (der dritte Bt) gehört dann konsequenterweise der Stufe 9 an. Es ergeben sich ca. 300.000 Jahre BP als Entstehungszeit für den dritten Bt. Sollten keine größeren Hiaten in der Schichtenfolge existieren, könnte der vierte Bt-Horizont in das Römerhof-Interglazial (KLostermann 1995) gestellt und mit der Sauerstoffisotopenstufe 11 verknüpft werden.

\section{Tektonische Bewegungen im Mittel- und Ober-Pleistozän}

Im Jahre 1987 wurden von Hollmann Sondierbohrungen in der Umgebung der Ziegeleigrube Dreesen abgeteuft. Die Bohrungen hatten zum Ziel, den zweiten Bt der Grube Dreesen auszukartieren und festzustellen, ob dieser durch jüngere tektonische Bewegungen beeinflußt wurde.

Die Kartierung zeigte, daß die Oberfläche der Jüngeren Hauptterrasse südlich der Rheindahlener Verwerfung deutlich abgesunken ist. Die Verwerfung selbst ist nur leicht erosiv überprägt. Für die Oberfläche der Jüngeren Hauptterrasse ergibt sich im Raum Rheindahlen ein durchschnittlicher Versatzbetrag von $5 \mathrm{~m}$. Auffallend ist auch die deutliche $\mathrm{Zu}-$ nahme der Lößmächtigkeiten südlich der Rheindahlener Verwerfung. Offenbar war das Gebiet südlich 
des Sprunges bereits während der Aufwehung der ältesten Lösse ein deutliches morphologisches Tiefgebiet. Die tektonische Aktivität des Rheindahlener Sprunges dürfte folglich einen ihrer Höhepunkte zwischen Jüngerer Hauptterrassen-Zeit und Holstein-Interglazial gehabt haben.

Südlich parallel zur Rheindahlener Verwerfung verläuft ein weiterer Sprung, die „Dahmener Verwerfung". Dort ist die Hauptterrassen-Oberfläche um bis zu $2 \mathrm{~m}$ verworfen. Die eemzeitliche Parabraunerde wird dagegen von der Rheindahlener Verwerfung überhaupt nicht beeinflußt, während sie durch die Dahmener Verwerfung um $2 \mathrm{~m}$ versetzt wird. Das bedeutet, daß die Rheindahlener Verwerfung mit Sicherheit während und nach der Eem-Warmzeit nicht mehr aktiv war. Die Dahmener Verwerfung dagegen war während des Quartärs wohl nur nach der Entstehung der eemzeitlichen Parabraunerde aktiv. Die tektonische Aktivität hat sich in der Umgebung Rheindahlens im Laufe des Quartärs offenbar in südlicher Richtung verlagert.

\section{Schriftenverzeichnis}

BosinsKI, G (1966): Der paläolithische Fundplatz, Ziegelei Dreesen - Westwand. Bonner Jahrb. 166, 318-343; Bonn.

(1967): Die mittelpaläolithischen Funde im westlichen Mitteleuropa. Fundamenta A4, Köln/Graz.

- (1971): Late Middle Palaeolithic groups in north-western Germany and their relations to early Upper Palaeolithic industries. The origin of Homo sapiens. (Ecology and Conservations 3), 153 - 160.

- (1983): Die jägerische Geschichte des Rheinlandes Einsichten und Lücken. - Jahrb. d. Römisch-Germanischen Zentralmuseums Mainz 30, 81-112; Mainz.

- Kunst und Altertum am Rhein. Führer des Rheinischen Landesmuseums Bonn 118. Köln.

- (1993): Der Neandertaler und seine Zeit. Archäol. im Ruhrgebiet 1991, 45-48; Gelsenkirchen.

- \& BRUNNACKER, K. (1973): Eine neue mittelpaläolithische Fundschicht in Rheindahlen. Arch. Korrbl. 3, 1-6; Mainz.

BrockmeIER, H. (1929): Über Steinwerkzeuge aus der Gegend von Mönchengladbach. Berichte des Niederrheinischen Geol. Ver., 78-80; Mönchengladbach.

BRUNNACKER, K. (1966): Das Profil „Westwand“ der Ziegeleigrube Dreesen in Rheindahlen. Bonner Jahrb. 166, 344-356; Bonn.

- (1973): Geologie. In: G. Bosinski \& K. BrunNaCKer: Eine neue mitteläolithische Fundschicht in Rheindahlen, Arch. Korrbl. 3; Mainz.

Cahen, D. \& Haesaerts, P. (1984): Peuples chasseurs de la Belgique préhistorique dans leur cadre naturel., 149 ff; Bruxelles.

Hollmann, P. (1987): Kartierung der spätpleistozänen Sandüberdeckung der Lößablagerungen beiderseits des Rheindahlener Sprunges (Blatt 4704 Mönchengladbach). - 19 S. 4 Abb., 1 Tab., 4 Taf., 1 Anl.; Krefeld (unveröff. Prüfungsarbeit).

KAHRS, E. (1951): Die Gliederung des Lösses an Ruhr und Niederrhein und die Stratigraphie der Fundstelle Rheindahlen. Bonner Jb. 151, 47-49; Bonn.
Klostermann, J. (1985): Versuch einer Neugliederung des späten Elster- und des Saale-Glazials der Niederrheinischen Bucht. Geol. Jahrb. A 83: 3-42, 22 Abb., 1 Tab.; Hannover.

- (1990): Rheindahlen-Löß und Menschheitsgeschichte. - Geol. Kt. Nordrh. - Westf. 1:100 000, Erl., C 5102: 6775, 1 Abb., 1 Tab.; Krefeld.

- (1992): Das Quartär der Niederrheinischen Bucht. 200 S., 30 Abb., 8 Tab., 2 Taf.; Krefeld.

- (1995). Nordrhein-Westfalen. - In: BENDA, L.: Das Quartär von Deutschland: - INQUA 1995; Berlin (in Vorber.).

- \& Rehagen, H.-W., \& Wefels, U. (1988): Hinweise auf eine saalezeitliche Warmzeit am Niederrhein. - Eiszeitalter u. Gegenwart, 38: 115-127, 5 Abb., 1 Tab; Hannover.

NARR, K. J. (1951): Alt- und mittelpaläolithische Funde aus rheinischen Freilandstationen. Bonner Jahrb. 151, 547; Bonn.

NEuBert, A. (1984): Pleistozäne Deckschichten und rezente Böden als Einflußgrößen der Grundwasserneubildungsrate - dargestellt am Grundwassererneuerungsmodell 11 Mönchengladbach. Diplomarbeit, Ruhr-Universität Bochum.

PAAS, U. (1962): Rezente und fossile Böden auf niederrheinischen Terrassen und deren Deckschichten. Eiszeitalter u. Gegenwart, 12: 165-230, 32 Abb.; Öhringen/Württ.

PAHLKE, U. (1984): Geomorphologische und pedologische Untersuchungen am Linken Niederrhein - Ein Beitrag zur Ermittlung der Grundwasserneubildung. Diplomarbeit Ruhr-Universität Bochum.

SCHIRMER, W. \& FELdMANN, L. (1992): Das Lößprofil von Rheindahlen, Niederrhein. In: Arbeitskreis Paläopedologie (Hrsg.). Bodenstratigraphie im Gebiet von Maas und Niederrhein, 76-85. Deutsche Bodenkundl. Gesellsch. Kiel.

Schmitz, R.-W. \& Thissen, J. (1994): Das Acheuléen im Niederrheingebiet. In: L. Fiedler (Hrsg.): Das Acheuléen in Deutschland. Stuttgart (im Druck).

SCHÜTRuMPF, R. (1966): Pollenanalytische Untersuchungen in den Lößschichten von Rheindahlen. - Bonner Jb. 166: 357-358; Bonn.

Steusloff, U. (1951): Periglaziale Böden aus zwei Eiszeiten im Niederrheinischen Löß bei Rheindahlen. Niederrh. Jahrb. 3, 18-19.

Thieme, H. (1977): Altpaläolithische Funde in der Ziegeleigrube Dreesen in Rheindahlen, Stadtkreis Mönchengladbach. Arch. Korrbl. 7, 235-239; Mainz.

- (1980): Neue Untersuchungen zu paläolithischen Fundschichten in Mönchengladbach-Rheindahlen. Ausgr. im Rhld. 1979, 57-59; Köln.

- (1982): Die Ziegeleigrube Dreesen in Mönchengladbach-Rheindahlen - ein mehrschichtiger altsteinzeitlicher Fundplatz. Rheydter Jb. 14, 11-33.

- (1983 a): Der paläolithische Fundplatz Rheindahlen. Dissertationsschrift, Universität Köln.

- (1983 b): Mittelpaläolithische Siedlungsstrukturen in Rheindahlen (BRD), Ethnographisch-Archäologische Zeitschrift 24, 362-374.

- \& Brunnacker, K. \& Juvigné, E. (1981): Petrographische und urgeschichtliche Untersuchungen im Lößprofil von Rheindahlen/Niederrheinische Bucht. Quartär 31/32: 41-67, 12 Abb., 1 Tab.; Bonn.

Thissen, J. (1986): Ein weiterer Fundplatz der WestwandFundschicht (B 1) von Rheindahlen. Arch. Korrbl. 16, 111-121; Mainz. 
- (1988): Die Grabungsfläche 1984/85 in der WestwandFundschicht von Rheindahlen. Unveröff. Magisterarbeit, Universität Köln.

- (1989): Ein Fundplatz des Magdalénien am Linken Niederrhein bei Kamphausen, Gem. Jüchen, Kreis Neuss. Arch. Korrbl. 19, 315-323.

- (1990): Paläolithische Fundplätze in der Ziegeleigrube Dreesen in Mönchengladbach-Rheindahlen. Lößstratigraphie und Ökologie im Alt- und Mittelpaläolithikum Eurasiens. Symposium im Forschungsbereich Altsteinzeit des Römisch-Germanischen Zentralmuseums Mainz auf Schloß Monrepos, Neuwied.

- (1993): Das Mittelpaläolithikum vom Liedberg, Bonner Jahrb. 193, 221-235.

- (1994): Paläolithische und mesolithische Funde im Kreis Neuss. Fund und Deutung. Neuere archäologische Forschungen im Kreis Neuss. - Kreisheimatb. Neuss, 5: 13-42, 1 Taf.; Neuss.

Tuffreau, A., Révillion, S., Sommé, J., Aitken, M. J., HuXtaBLe, J. \& LeroI-Gourhan, A. (1985): Le gisement Paleo- lithique moyen de Seclin (Nord-France). Arch. Korrbl. 15, 131-138; Mainz.

- \& Almeloot-Van Der Hejjden, N. (1990): Der mittelpaläolithische Fundplatz von Riencourt-les-Bapaume (Nord-Frankreich). Lößstratigraphie und Ökologie im Alt- und Mittelpaläolithikum Eurasiens. Symposium im Forschungsbereich Altsteinzeit des Römisch-Germanischen Zentralmuseums Mainz auf Schlors Monrepos, Neuwied.

VÉRTES, L. (1965): Typology of the Buda industry. A pebbletool industry from the Hungarian Lower Palaeolithic. Quaternaria 7, 185-195.

WichtMANN, H. (1987): Bodenphysikalische Untersuchungen zur quartärgeologischen Deutung der Lößprofile Dreesen und Dahmen in Mönchengladbach-Rheindahlen (Archiv des GLA NRW, Krefeld).

Manuskript eingegangen am 28. 12. 1993. 

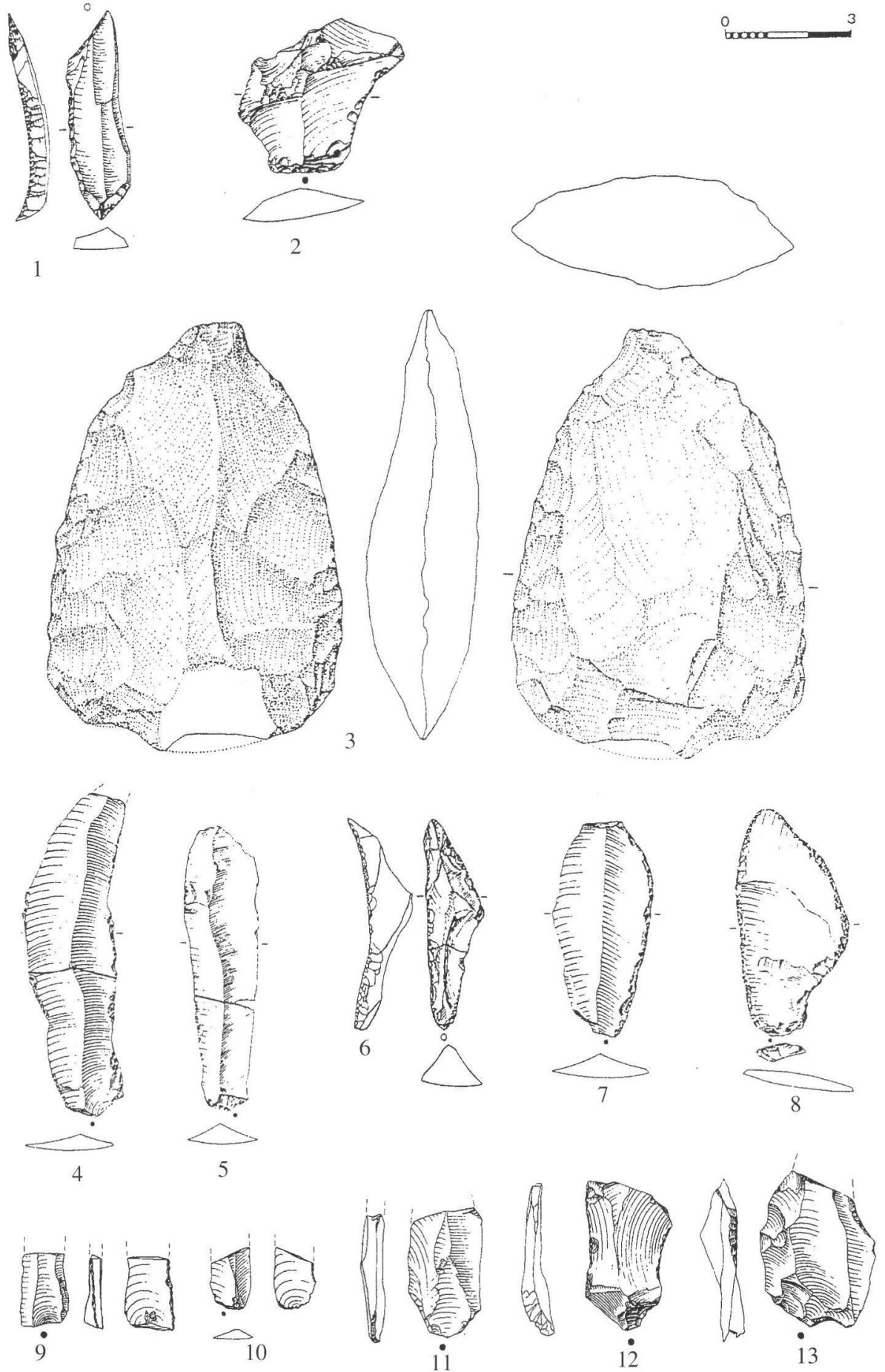

Taf. 1: Mönchengladbach-Rheindahlen: 1. Geknickte Rückenspitze (A 1) - 2. Retuschierter Abschlag (A 1) - 3. MTA-Keil aus Quarzit (A 3) - 4. - 8. Retuschierte Formen der Grabung 1964/65 (B 1/B 2) - 9. - 13. Retuschierte Formen der Grabung 1984/85 (B 1/B 2), 1-2: Thieme 1982; 3: Thieme, Brunnacker \& Juvigné 1981; 4-8: Thieme 1982; 9-13: Tilissen 1986 

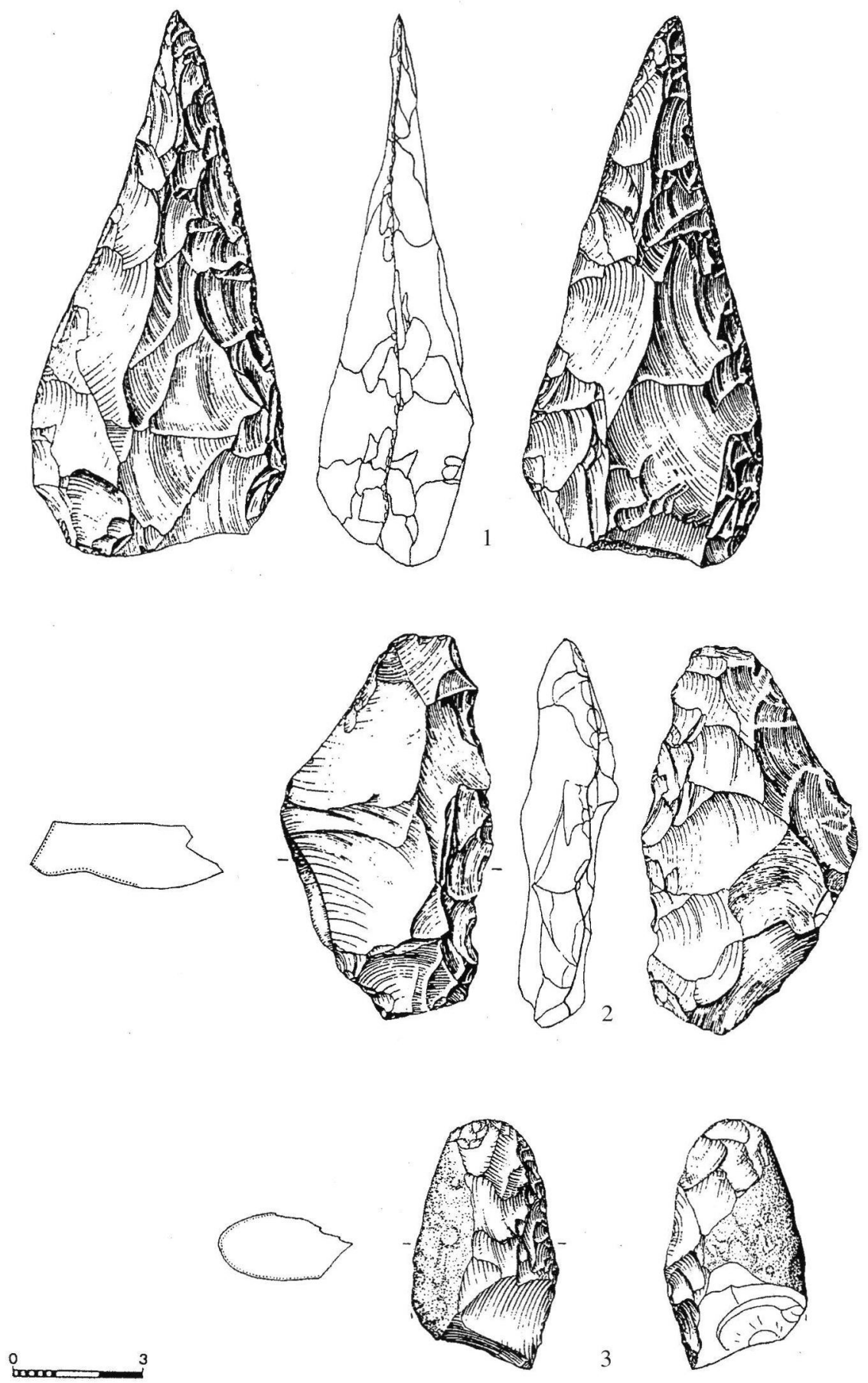

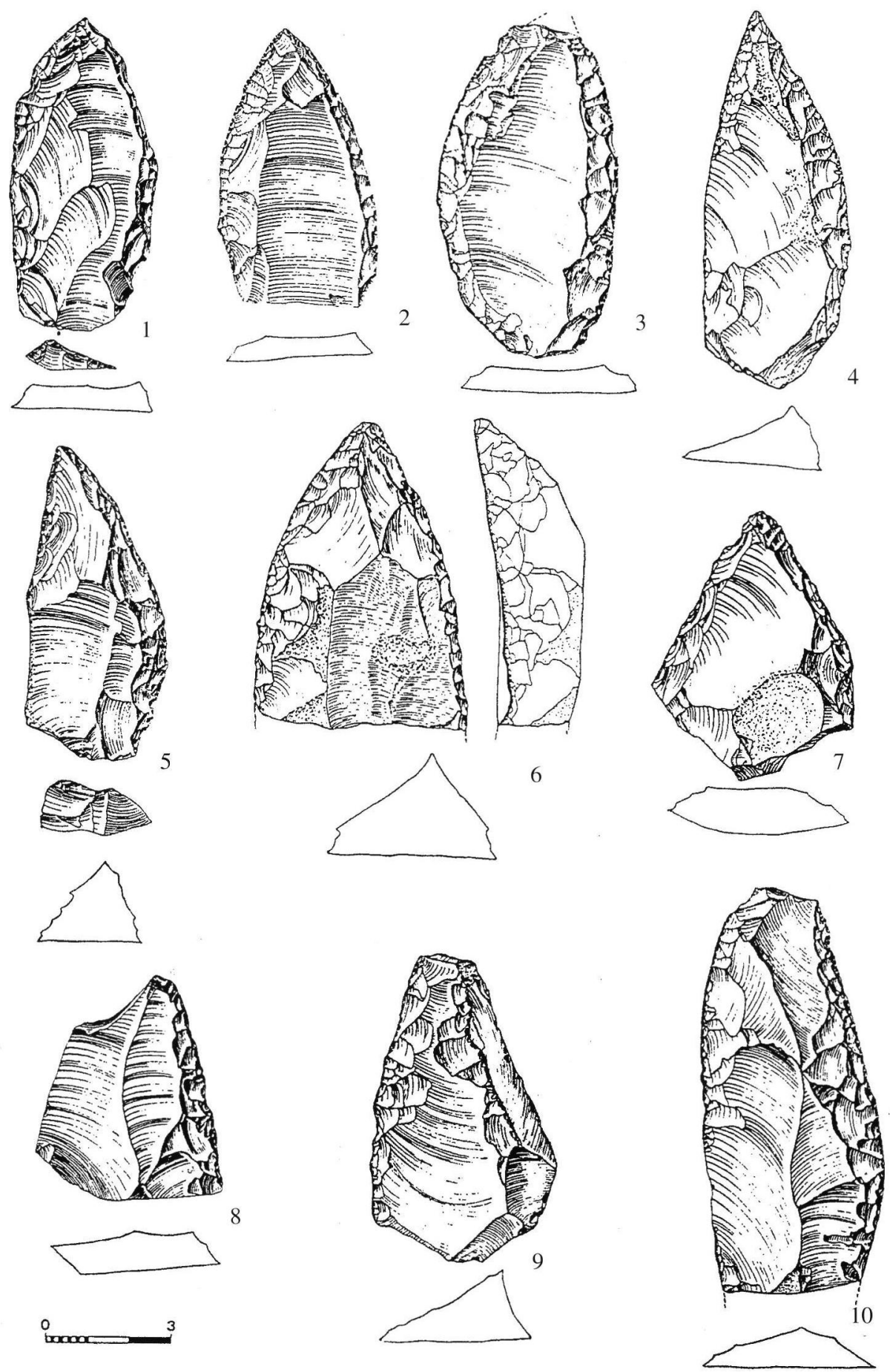

Taf. 3: Mönchengladbach-Rheindahlen: 1 - 5. Konvexe Spitzen - 6. Bruchstück einer Limace (?) - 7. Spitzschaber - 8. 9. Einfache Schaber - 10. Retuschierte Klinge. - Alle B 3. 1 - 10: BOSINSKI 1967 

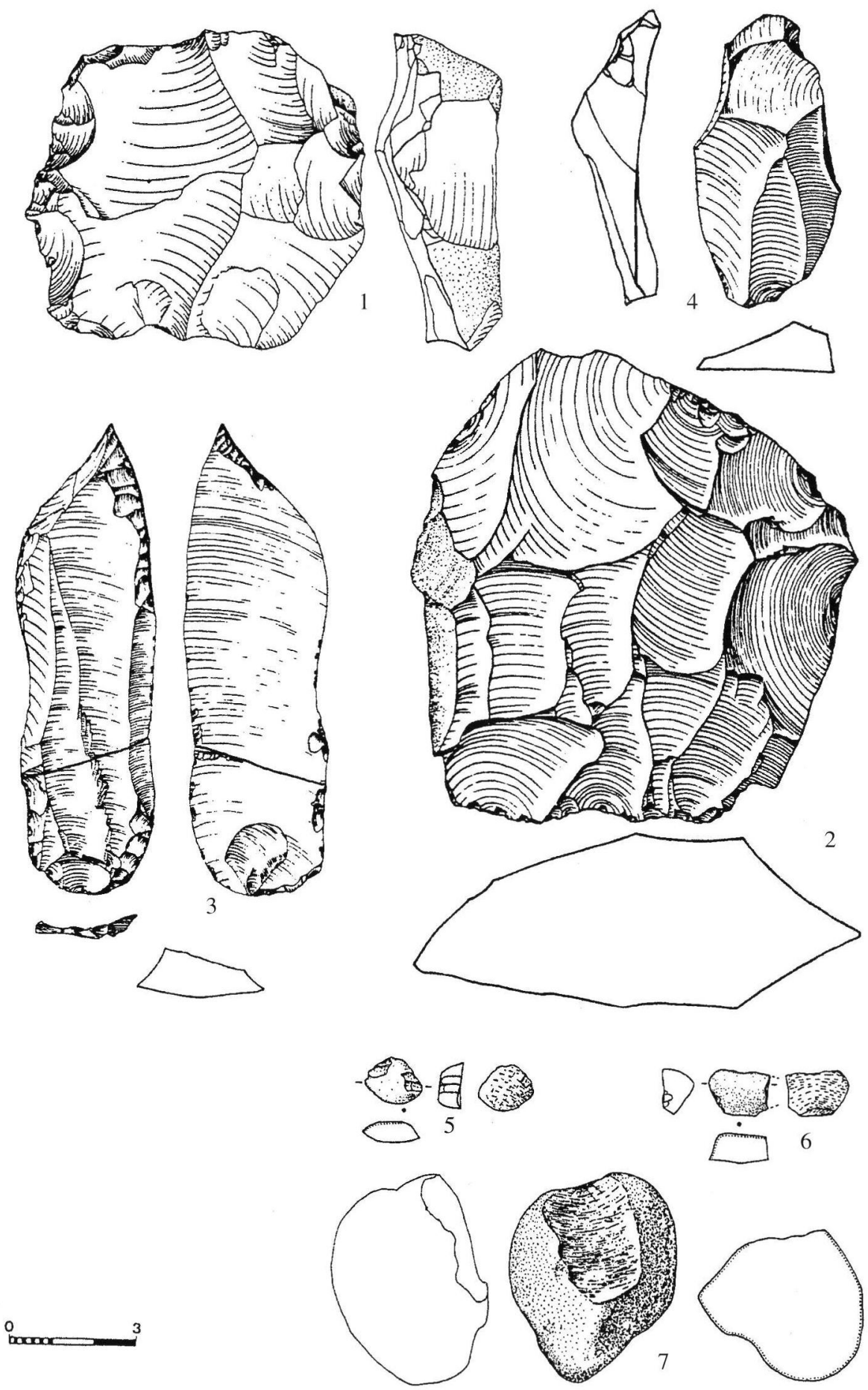